\title{
Sistema experto para análisis de falla de ejes
}

\author{
V.H. Jacobo-Armendáriz, S.M. Cerrud-Sánchez, M.A. Ramírez-Rodríguez, A. Ortiz-Prado \\ Departamento de Ingeniería Mecánica \\ Facultad de Ingeniería, UNAM \\ E-mails:vjacobo@dimei.fi-c.unam.mxycerrud@servidor.unam.mx
}

(recibido: julio de 2001; aceptado: diciembre de 2001)

\begin{abstract}
Resumen
Con el fin de contar con un medio eficaz para la solución de problemas relacionados con el análisis de falla, en donde la experiencia juega un papel fundamental, el Departamento de Ingeniería Mecánica de la Facultad de Ingeniería de la UNAM, está desarrollando el proyecto "Sistema experto para análisis de falla de elementos mecánicos metálicos", dicho sistema busca resolver el inconveniente de la escasez de expertos y se orienta, tanto a las necesidades de la industria metalmecánica como a las de la docencia. Presenta también complicaciones en su desarrollo, ya que se pretende la posibilidad de realizar análisis de falla de elementos como ejes, engranes, pernos, bielas, resortes, tornillos etc. Por lo anterior, se estableció como estrategia el desarrollo del sistema por módulos, ya que teniendo la experiencia de elaboración de un primer módulo, la construcción de los siguientes se facilitaría. En este artículo se presenta el desarrollo de un primer módulo orientado a la solución de problemas de ejes, mismo que servirá como plataforma para la expansión de la base de conocimientos del sistema global. Este módulo de ejes se desarrolló dentro de la estructura de lo que será el sistema global, incluyendo un programa auxiliar para la determinación de esfuerzos máximos.
\end{abstract}

Descriptores: sistemas expertos, análisis de falla, diseño mecánico, ejes.

\section{Abstract}

An expert system for the failure analysis in metallic mechanical devices is being developed at the Mechanical Engineering departaments of the Faculty of Engineering of the UNAM to provide a solution for the failure analysis in cases where considerable experience in needed. The system pretends to resolve the scarceness of experts in the field of failure analysis and is oriented toward the metal-mechanics industry and will also have didactic applications. The main compliction in the development of the system is the broad range of applications envisioned, like gears, shafts, bolts and springs between others. Therefore, it was decided to develop the system in a modular way, using the experience obtained in a first module to proceed faster in the others. The present paper presents the elaboration of the module that analyses shafts. This will serve as the base to expand the system toward other components. It uses the general structure of the global system, including an auxiliary software for the determination of maximum stresses in the corresponding parts.

Keywords: expert systems, failure analysis, mechanical design, shafts. 
DOI: http://dx.doi.org/10.22201/fi.25940732e.2002.03n3.012

\section{Introducción}

El Departamento de Ingeniería Mecánica de la Facultad de Ingeniería a través de la Unidad de Investigación y Asistencia Técnica en Materiales, ha desarrollado una línea de trabajo en torno a sistemas inteligentes para análisis de falla, en particular, se ha enfocado a la creación del sistema experto para análisis de falla de elementos mecánicos metálicos (SEAFEM). La idea fundamental es contar con un sistema de cómputo en donde esté confinado el conocimiento de expertos en análisis de falla (AF) y de casos reportados en la literatura (ASM, 1974; 1986 y 1987). Una vez construido el sistema es factible su reproducción, por lo que las empresas podrán contar con el soporte tecnológico sin la necesidad de que el experto esté presente. La orientación del SEAEFM, es la de actuar como colega, facilitando la toma de decisiones, a la vez de servir como soporte tecnológico a las pequeñas, medianas y grandes empresas; las cuales generalmente en México no disponen de personal capacitado en el área. El sistema se caracterizará por aprender de su propia experiencia, garantizando así, su crecimiento y confiabilidad. Este sistema podrá ser operado por personas con estudios de ingeniería, y proporcionará ayuda al analista para tomar decisiones relacionadas al AF. Además, contiene un módulo en donde se cuantifican los esfuerzos máximos a los que estuvo sometido el elemento en el momento de servicio, así como la ubicación de dichos esfuerzos. Por otro lado, el sistema no sólo ayuda a resolver un problema de AF, sino que puede utilizarse como tutorial para capacitar a personal en esta área.

La justificación para el desarrollo del SEAFEM (Cerrud, et al. 1998), se establece no sólo por lo expresado en líneas anteriores, sino que además se tomaron en cuenta el procedimiento de evaluación de aplicaciones de sistemas propuesto por Beckman, y los desarrollos que sobre el tema se han llevado a cabo en algunas instituciones, por ejemplo, bajo los auspicios de sociedades como la NACE (National Association of Corrosion Engineers) se han realizado SE para el AF, por corrosión (Roberge, 1996). En algunas universidades (Southampton, U.K.) Graham y Mellor (1997a) y (1997b), bajo otro enfoque, reportan avances en el desarrollo de sistemas expertos (SE) (Walton, 1991) para AF.

\section{Objetivos del SEAFEM}

Para el desarrollo del sistema se plantearon los siguientes objetivos:

1. Diseñar e implementar un sistema experto de tipo colega para la determinación de las causas de falla de elementos mecánicos metálicos.

2. Construir el sistema bajo ambiente PC-Windows para facilitar su aplicación.

3. Determinar las acciones correctivas requeridas o propuestas.

4. A partir de la imagen digitalizada, el sistema indicará las probables condiciones de carga que condujeron a la falla

5. Ahorrar tiempo en el AF.

6. Contar con un experto que se pueda consultar en diferentes lugares a la vez.

\section{Metodología para el desarrollo del SEAFEM}

A continuación, se presentan los diferentes pasos que se han aplicado para la construcción del sistema:

1. Desarrollo del ingeniero del conocimiento

2. Integración del grupo de expertos en AF.

3. Establecimiento de las características de operación del SE.

4. Adquisición del conocimiento.

5. Estructuración y representación del conocimiento.

6. Construcción de la base de conocimientos.

7. Desarrollo de un subprograma para el cálculo de esfuerzos máximos.

8. Selección del lenguaje o la herramienta de desarrollo.

9. Construcción del prototipo.

10. Construcción de la interfase con el usuario.

11. Verificación y validación del prototipo.

12. Expansión de la base de conocimientos del prototipo. 
13. Verificación y validación del sistema.

14. Integración del sistema.

15. Desarrollo e integración de módulos auxiliares de análisis de esfuerzos, deterioro ambiental, propagación de grietas, etc.

16. Verificación y validación del sistema.

\section{Avances del SEAFEM}

Actualmente el proyecto ha concluido en su totalidad los puntos $1,2,3,7,8$ y 10 . Los puntos 4 , $5,6,9$ y 11 muestran avances parciales.

\section{Problemática general del desarrollo del SEAFEM (Cerrud et al., 2000)}

La fase de adquisición del conocimiento es la más conflictiva, pues demanda entre un 60 y $70 \%$ del tiempo de desarrollo, y al ser la primera fase del proceso, es una actividad neurálgica. Por otro lado, en el AF el conocimiento que se involucra para el análisis y la toma de decisiones es muy complejo, razón por la que se debe definir un método que permita la extracción, selección, estructuración, organización y validación del conocimiento de manera confiable, ya que la forma en que se realice, repercutirá en las fases subsecuentes del desarrollo del sistema. Independientemente de lo anterior, los expertos no tienen el tiempo necesario para involucrarse de lleno en el proyecto.

La representación del conocimiento es otro problema, ya que para trasladar el conocimiento del experto a una computadora es necesario utilizar estructuras discretas que indican complicaciones al verterlo. Las estructuras que se plantearon fueron las reglas de decisión. En el caso concreto del AF es factible desarrollar una estructura de reglas de este tipo. La práctica general del AF parte de conocer ciertas premisas, éstas a su vez son suficientes para encontrar algunas conclusiones intermedias que vayan delimitando la solución del problema. Dado que la solución de un caso de AF es factible que posea varias respuestas tentativas, un SE se convierte en una herramienta idónea que puede dar solución a la disyuntiva que éstas representan.
Determinar las causas de falla en elementos sometidos a cargas uniaxiales es sencillo, ya que las características de las zonas de falla son fácilmente identificables. En las situaciones donde los elementos mecánicos se someten a solicitaciones triaxiales, además de condiciones dinámicas de carga, variaciones de temperatura, desgaste y corrosión, la determinación de la causa de falla se vuelve compleja, requiriendo de un grupo interdisciplinario coordinado por un experto en AF

En principio, la estructuración de las reglas que representan el conocimiento del AF están enfocadas a determinar las características intrínsecas de la falla, principalmente el tipo de falla que se presenta; la función principal y la más deseable del SE para AF, es determinar las condiciones que provocaron la falla. Así, fue necesario encontrar y desarrollar reglas que involucraran los diferentes tipos de falla y demás información contenida en ellas, con condiciones que activarán a éstas. Esto es una tarea compleja, ya que existen dentro del AF condiciones diversas que conducen a la determinación de no sólo un tipo de falla, existen fallas en las que varios mecanismos de fractura estuvieron involucrados y la superficie contiene características de todos estos mecanismos, además de diversas condiciones de operación que promueven la presencia de un tipo de falla en particular. Sin embargo, aunque la representación del conocimiento involucrado en el AF hace de ésta una tarea compleja, mas no imposible.

\section{Estrategia para el desarrollo del sistema global}

El SEAFEM es un sistema que busca dar solución a problemas relativos al AF de diversos elementos mecánicos como ejes, pernos, engranes, bielas etc., por lo que inicialmente se estructuró el sistema desde una visión global. También se diseñó la interfase de usuario y la estructura de la base de conocimientos de acuerdo con este planteamiento. Si bien es cierto que ésta es la base estructural del sistema, buscar construirlo como un todo resultó problemático y se manifestó en el propio desarrollo del prototipo (Jacobo et al., 
DOI: http://dx.doi.org/10.22201/fi.25940732e.2002.03n3.012

2000), ya que debido a lo robusto del sistema todas las acciones se complicaron. Por lo anterior, se planteó como estrategia la construcción del sistema módulo por módulo, ya que teniendo la experiencia de este primer subsistema, los demás se facilitarían por tener la experiencia sobre los inconvenientes que se pudieran presentar en la adquisición y representación del conocimiento. Debido a la cantidad de información con la que se dispone y de la experiencia de solución de casos prácticos, el primer módulo que se desarrolló fue el relativo a ejes mecánicos metálicos. La estructura y desarrollo de la base de conocimientos, se realizó a partir de lo que indica la técnica de la matriz de experiencia para AF (Dieter, 1991), ya que establece un criterio que organiza la información de la base tomando en cuenta tres aspectos: tipos de falla, modos de falla y conclusiones. A continuación se presenta la forma de construcción de este módulo y la operación del mismo, finalmente se establecen las recomendaciones para el desarrollo de otros módulos necesarios para la integración del sistema global.

\section{Desarrollo del sistema experto para análisis de falla de ejes}

El enfoque inicial para su construcción fue desarrollar un módulo experimental con base en los datos proporcionados por la inspección visual del usuario, con el fin de determinar de forma sencilla, a través de una interfase con elementos gráficos, el tipo de falla que se presenta en los ejes. La base de reglas para este caso no es muy amplia (Figuras 1 y 2), dado que la información requerida para llegar a las conclusiones a partir de la inspección visual no es abundante. Sin embargo, mediante esta primera aproximación fue posible darse cuenta de la necesidad de encontrar una estructura lógica para el desarrollo de los cuestionamientos por parte del sistema hacia el usuario, de manera que existiese una secuencia que orientará al usuario en forma amigable y no contradictoria, evitando caer en cuestionamientos redundantes o repetitivos en el desarrollo de las preguntas. Con la lógica planteada, se estructuró la secuencia de operación del sistema global, que implica tanto el orden en que aparecen las pantallas de interacción con el usuario, como el de la activación de las reglas de decisión correspondientes.

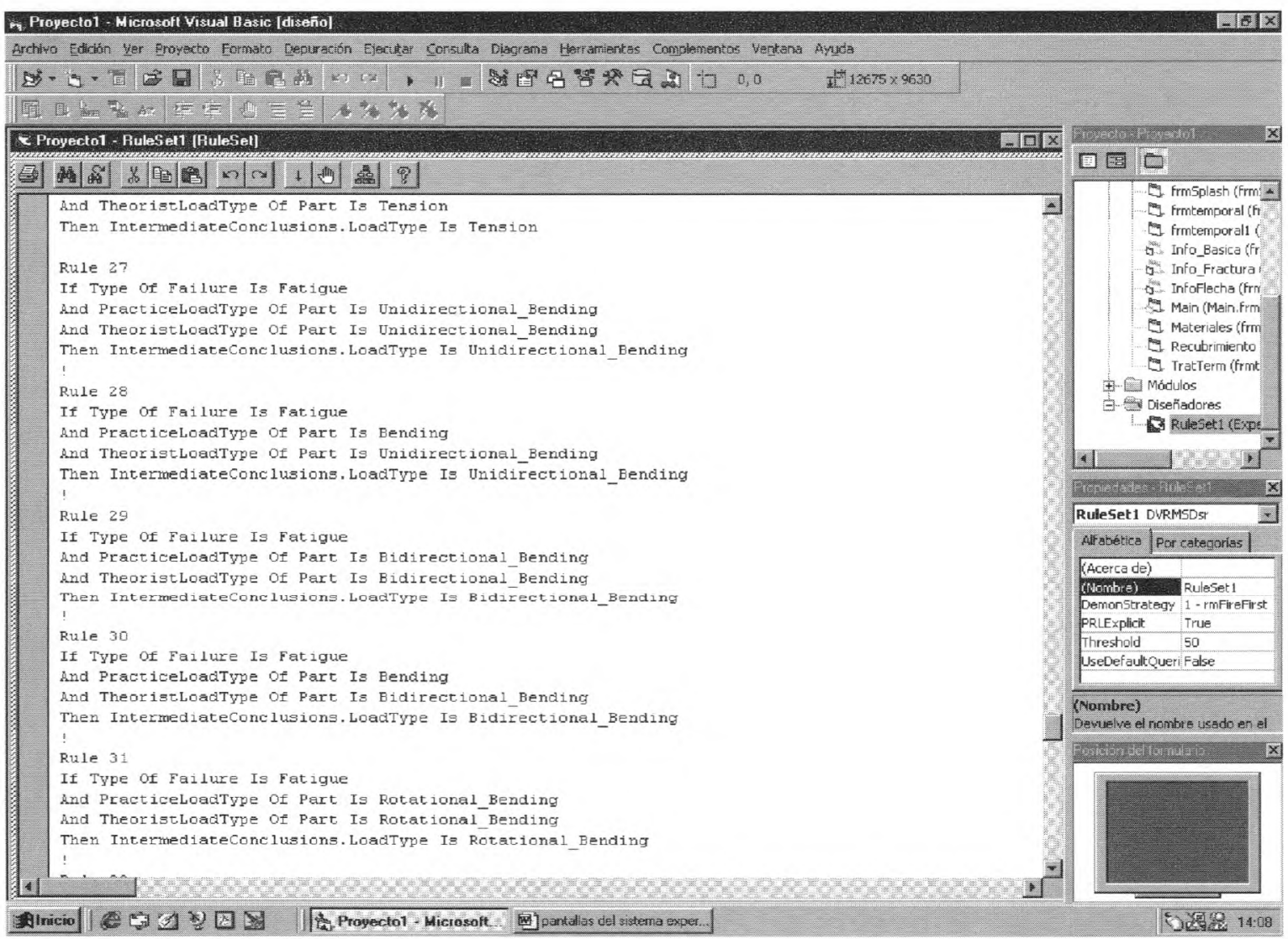

Figura 1. Reglas de fallas en ejes 


\section{Operación general del sistema global}

Tomando en cuenta la necesidad de establecer una jerarquía en la secuencia de operación del sistema, al principio se plantearon tres preguntas relevantes, que de manera conjunta aportan una gran cantidad de información sobre el elemento en estudio, éstas son referentes a:

\section{Material \\ Tipo de pieza \\ Sistema al que pertenece}

A través de estos conceptos se establece un marco característico de tres condiciones simultáneas, pero éste no es suficiente para obtener detalles específicos sobre la falla. Es a partir de esta información donde se inicia la ramificación del árbol de preguntas a través de la interacción con las pantallas, mismo que está relacionado con el árbol de reglas contenido en la base de conocimientos (Figura 2).

Como se ha mencionado, el sistema se desarrolló para el caso de ejes, sin embargo, al estar corriendo el programa sobre la plataforma del sistema global, aparecen una serie de opciones para el usuario: ejes, engranes, resortes, pernos, mecanismos $4 \mathrm{R}$, carcazas, etc., así el usuario elige la pieza en estudio. Una vez capturado el tipo de pieza, se define el sistema al que pertenece, y en este rubro, se localizan sistemas de transmisión par-potencia, generación de energía, almacenamiento de energía, soporte y los que se agreguen. Estas clasificaciones orientan hacia la determinación de las posibles causas, requiriéndose preguntas más específicas y, por ende, bifurcaciones del árbol. Posteriormente, surgen en la operación del sistema una serie de preguntas en un segundo plano, pero ligadas a las respuestas obtenidas a partir del primer nivel de preguntas. En el caso de ejes, estas cuestiones estarán relacionadas con características físicas, tipos de carga, geometría (sección transversal, continuidad en el diámetro, muescas, etc). De este modo se dan detalles cada vez más finos de la pieza, lo cual, logra mejores aproximaciones hasta obtener el resultado final. Terminada la inspección visual se hacen una serie de preguntas correspondientes a los parámetros básicos de diseño, información que será utilizada para el módulo de cálculo de esfuerzos. Los resultados de este módulo serán comparados con la información correspondiente al tipo de material y condiciones de operación almacenadas. Una vez definida la solución, el sistema buscará casos similares en la base de casos, validando o desechando el usuario, la decisión tomada.

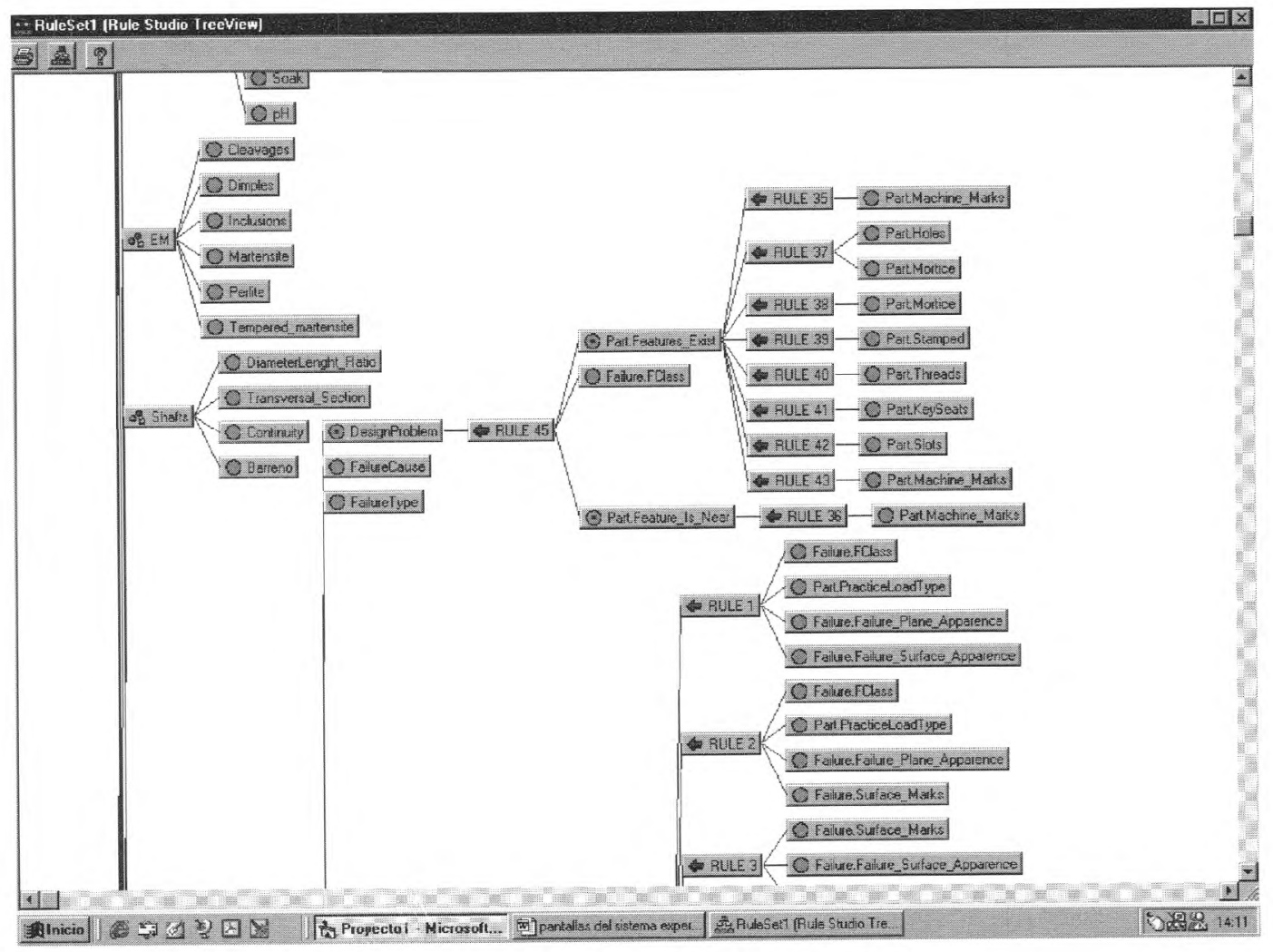

Figura 2. Estructura de árbol de reglas 
DOI: http://dx.doi.org/10.22201/fi.25940732e.2002.03n3.012

\section{Operación del sistema experto para ejes}

La secuencia de operaciones que sigue el usuario en su interacción con el sistema para solucionar un problema de análisis de falla de ejes es la siguiente:

1. Al inicio, el sistema cuestiona al usuario con relación al tipo de material y elemento mecánico. La interfase utilizada es del tipo de documento múltiple, lo cual facilita el movimiento a través de los distintos módulos por un menú principal. Se cuenta con la opción de elegir entre una gran gama de aceros y elementos mecánicos como ejes, pernos, engranes, etc.

2. Una vez que se seleccionó el elemento eje, se activa la siguiente pantalla (Figura 3) en donde se solicita información relativa al tipo de falla, maquinado, acabados, aspecto general de la pieza, condiciones de servicio y presencia de concentradores de esfuerzos. Esta pantalla funciona para cualquier elemento, puesto que almacena información genérica en donde el único cambio que existe es el icono del elemento analizado.

3. Si se activa la opción de concentradores de esfuerzos y se están analizando ejes, aparece una nueva ventana que contiene información sobre los distintos tipos existentes de concentradores para este elemento (Figura 4), de esta manera se puede cuantificar el coeficiente de seguridad para el diseño.

4. Una vez que se precisa la información requerida por la ventana principal (aspectos relativos a las condiciones de servicio y parámetros de diseño) se activa una ventana adicional en donde se captura información específica (geometría, dimensiones y condiciones de carga) relacionada con las condiciones de servicio de ejes (Figura 5).

5. En la siguiente ventana (Figura 6) se solicita información relativa al aspecto de la zona de fractura (textura, apariencia, marcas), asimismo, se demandará información al usuario con respecto al tipo de carga. Con esta información se determina la primera conclusión de la inspección visual (Figura 7).

6. En la figura 8 se presenta un ventana que se activa cuando la conclusión que se obtuvo en el punto anterior fue que la falla del eje se debería a fatiga, por lo que se presentan una serie de imágenes que muestran los distintos patrones de fractura causados por fatiga, dependiendo del tipo de carga y la concentración de esfuerzos; buscando con esto corroborar el estado de esfuerzos y el tipo de carga suministrados por el usuario anteriormente en la forma de información visual (Figura 6).

7. En la siguiente ventana (Figura 9), de acuerdo con la información obtenida en el punto anterior se establece una deducción con base en el tipo de carga y su relación con la superficie de fractura.

8. Existe un módulo auxiliar orientado hacia el cálculo de esfuerzos (Figura 10), el objetivo de este módulo es encontrar la magnitud de los esfuerzos principales y su ubicación en el elemento, para así poder comparar esta información con la recopilada por el usuario.

9. Se cuenta con un módulo adicional en donde el usuario puede comparar el caso resuelto con los contenidos, en una base de casos sobre fallas en ejes (Figura 11); esta rutina le sirve para tener información adicional y validar las conclusiones obtenidas por el sistema experto. 
DOI: http://dx.doi.org/10.22201/fi.25940732e.2002.03n3.012

V.H. Jacobo-Armendáriz, S.M. Cerrud-Sánchez, M.A. Ramírez-Rodríguez, A. Ortiz-Prado

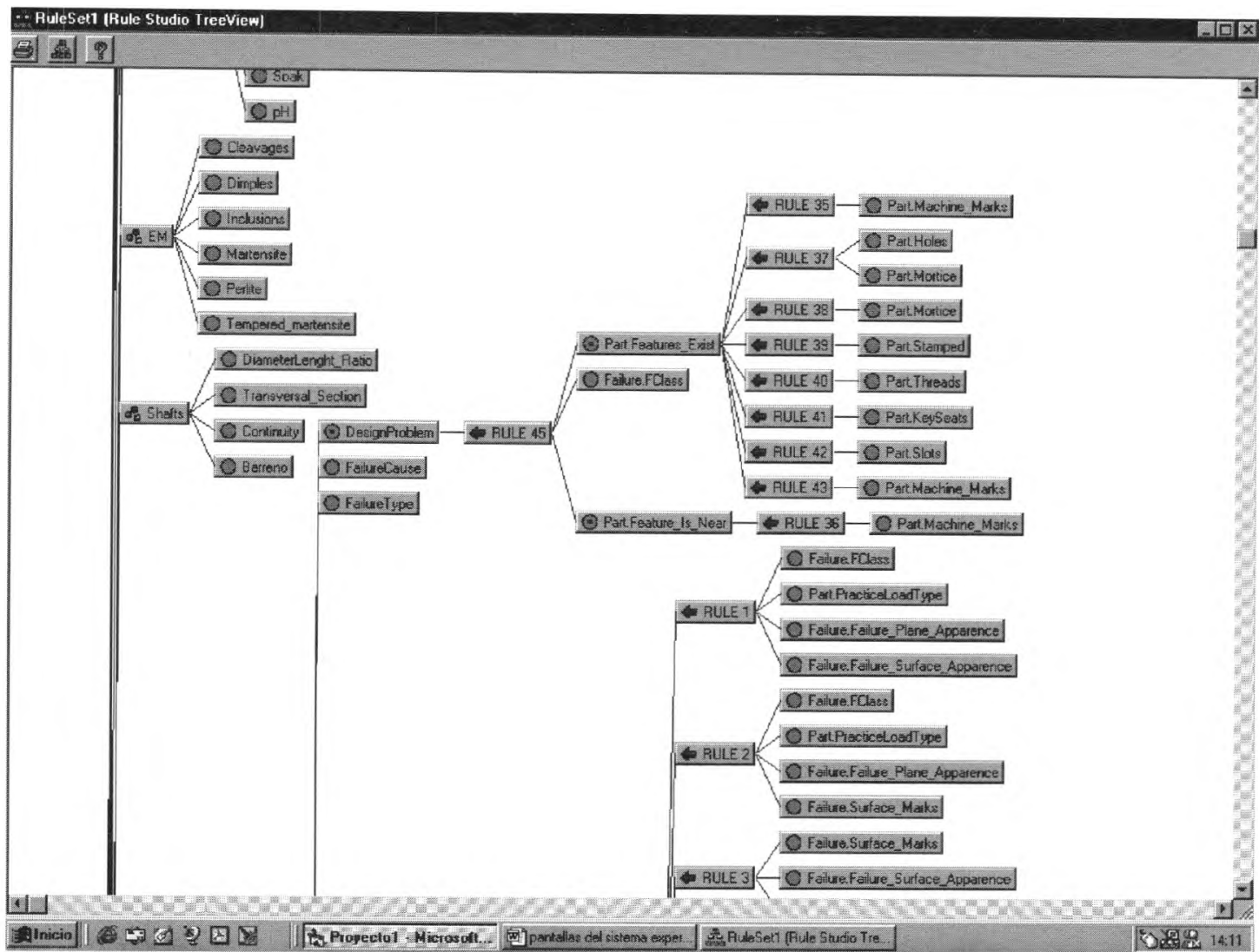

Figura 3. Información general sobre elementos mecánicos

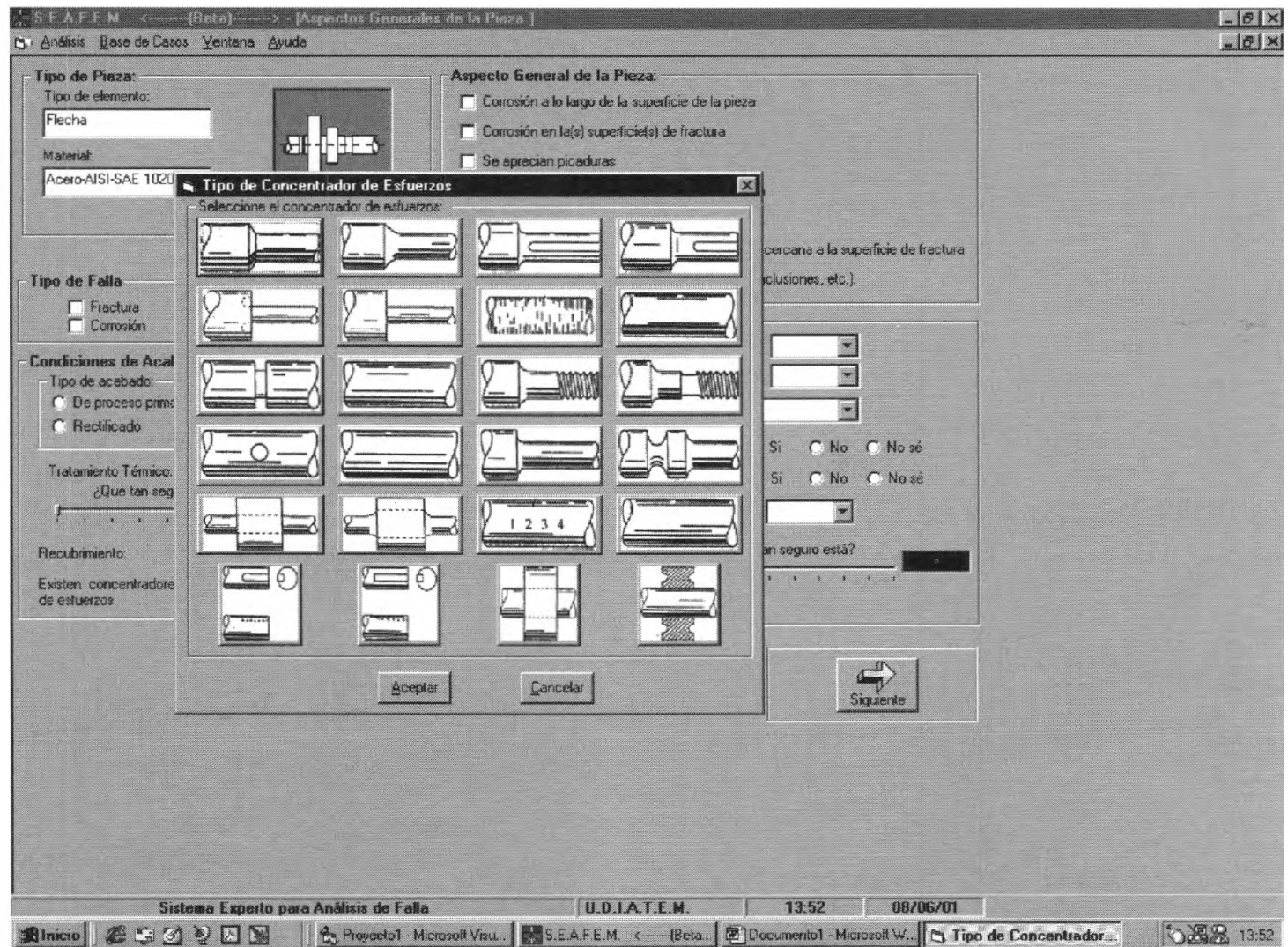

Figura 4. Opciones de concentradores de esfuerzos sobre ejes 
DOI: http://dx.doi.org/10.22201/fi.25940732e.2002.03n3.012

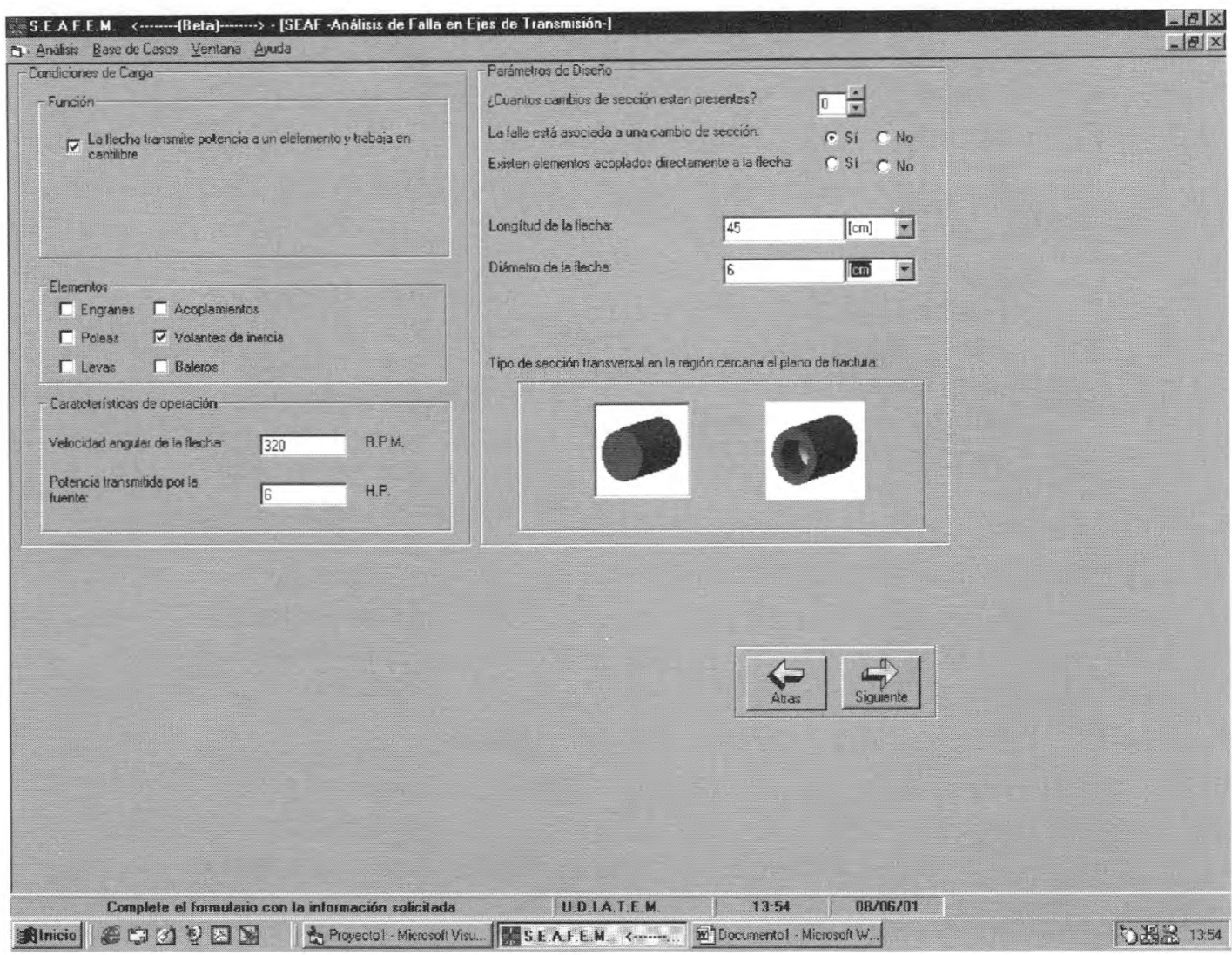

Figura 5. Información sobre condiciones de servicio de ejes

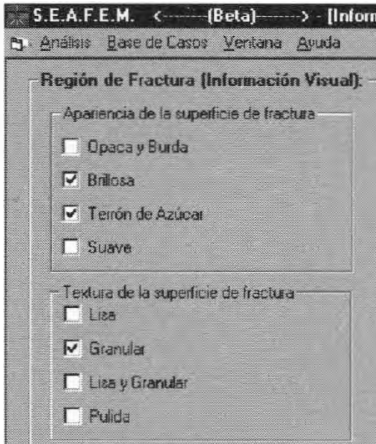

ormación Relacionada con la Fractura]

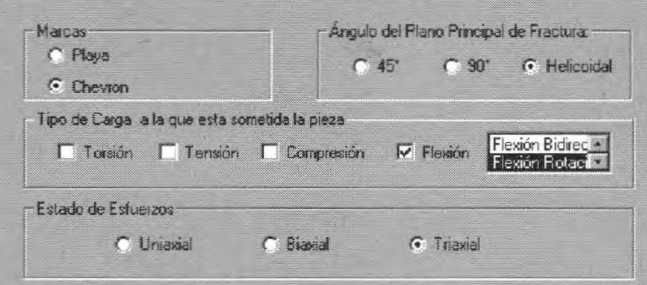

\section{T) Existen Cargas Cidicare}

T. Se aprecia una detormación apseciabte carcana a la regibin de fe hacturs

$\Gamma$ La fractura se encuentra sobre el concentuador de esfuerzos 
DOI: http://dx.doi.org/10.22201/fi.25940732e.2002.03n3.012

V.H. Jacobo-Armendáriz, S.M. Cerrud-Sánchez, M.A. Ramírez-Rodríguez, A. Ortiz-Prado

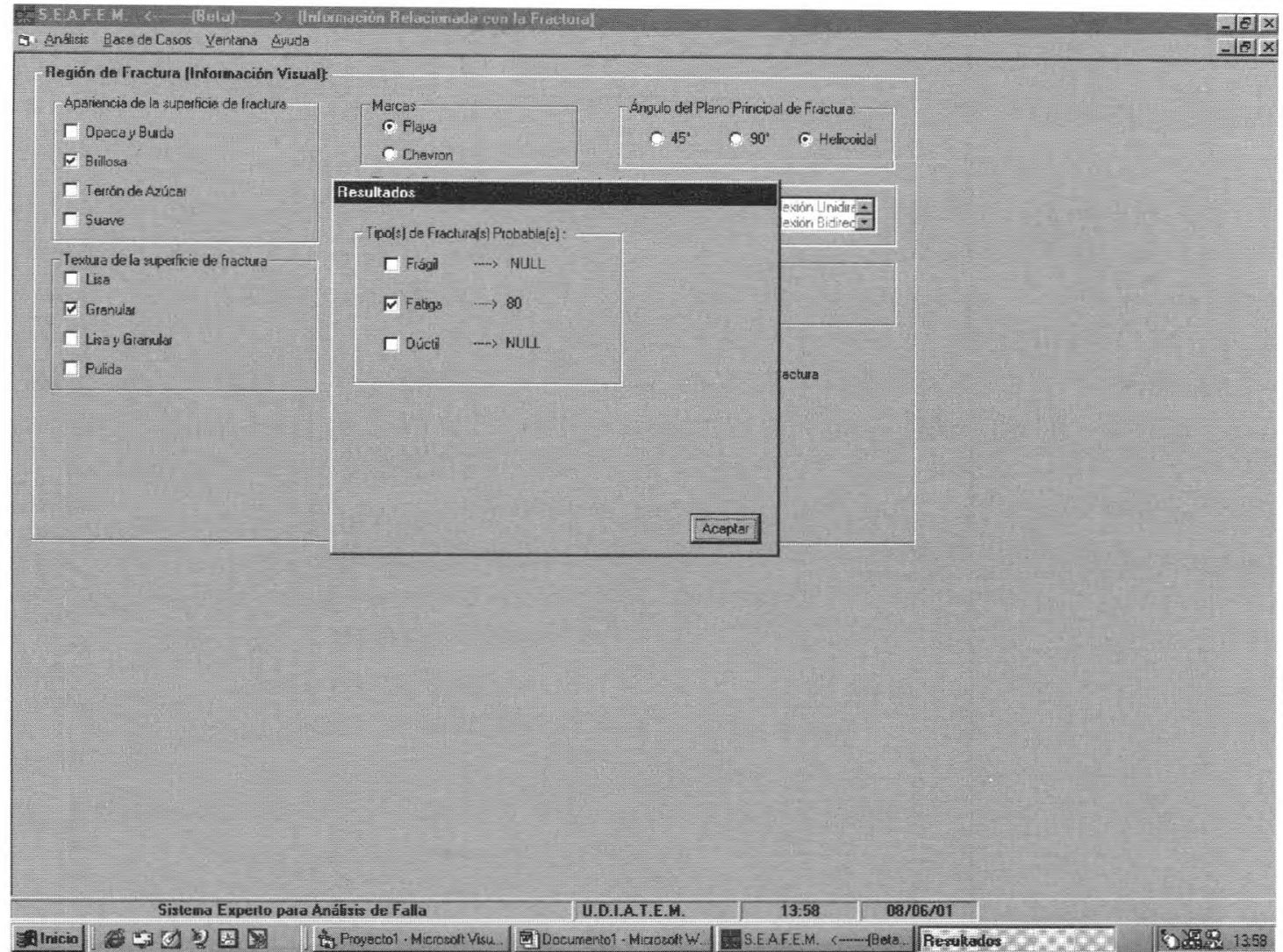

Figura 7. Conclusiones de la inspección visual

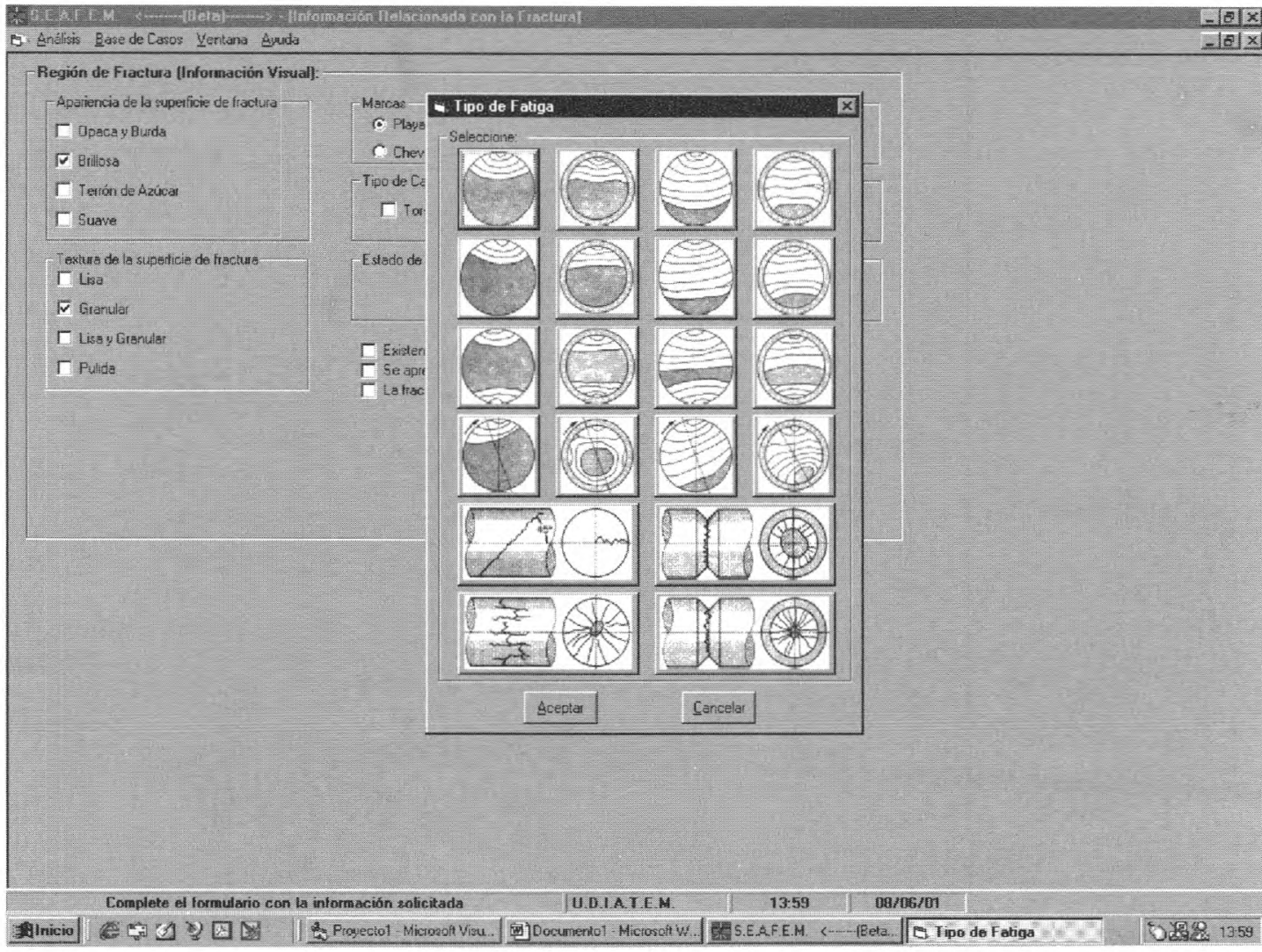

Figura 8. Muestrario de probetas con fracturas por fatiga a diferentes condiciones de carga 
DOI: http://dx.doi.org/10.22201/fi.25940732e.2002.03n3.012

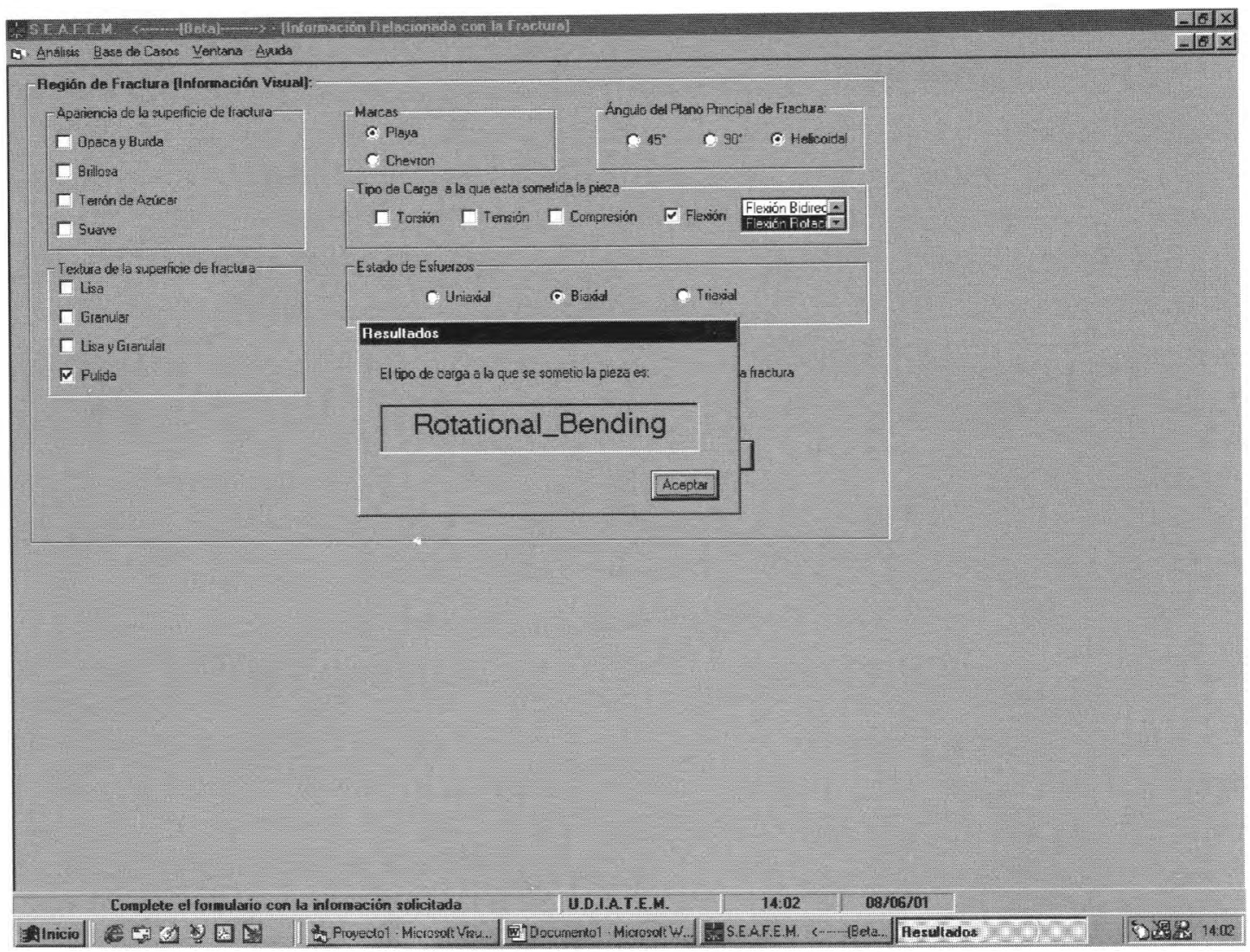

Figura 9. Deducción sobre el tipo de carga

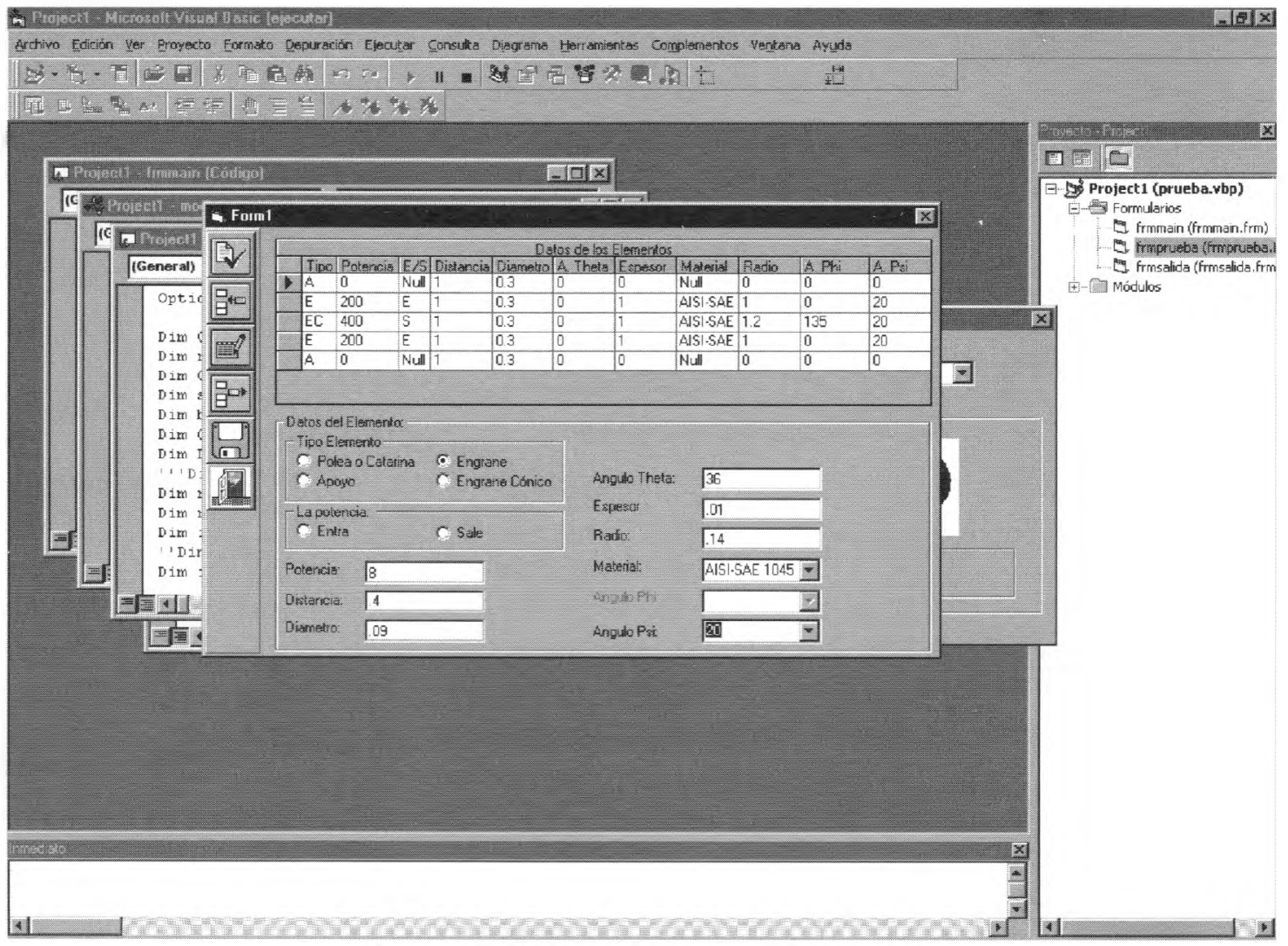




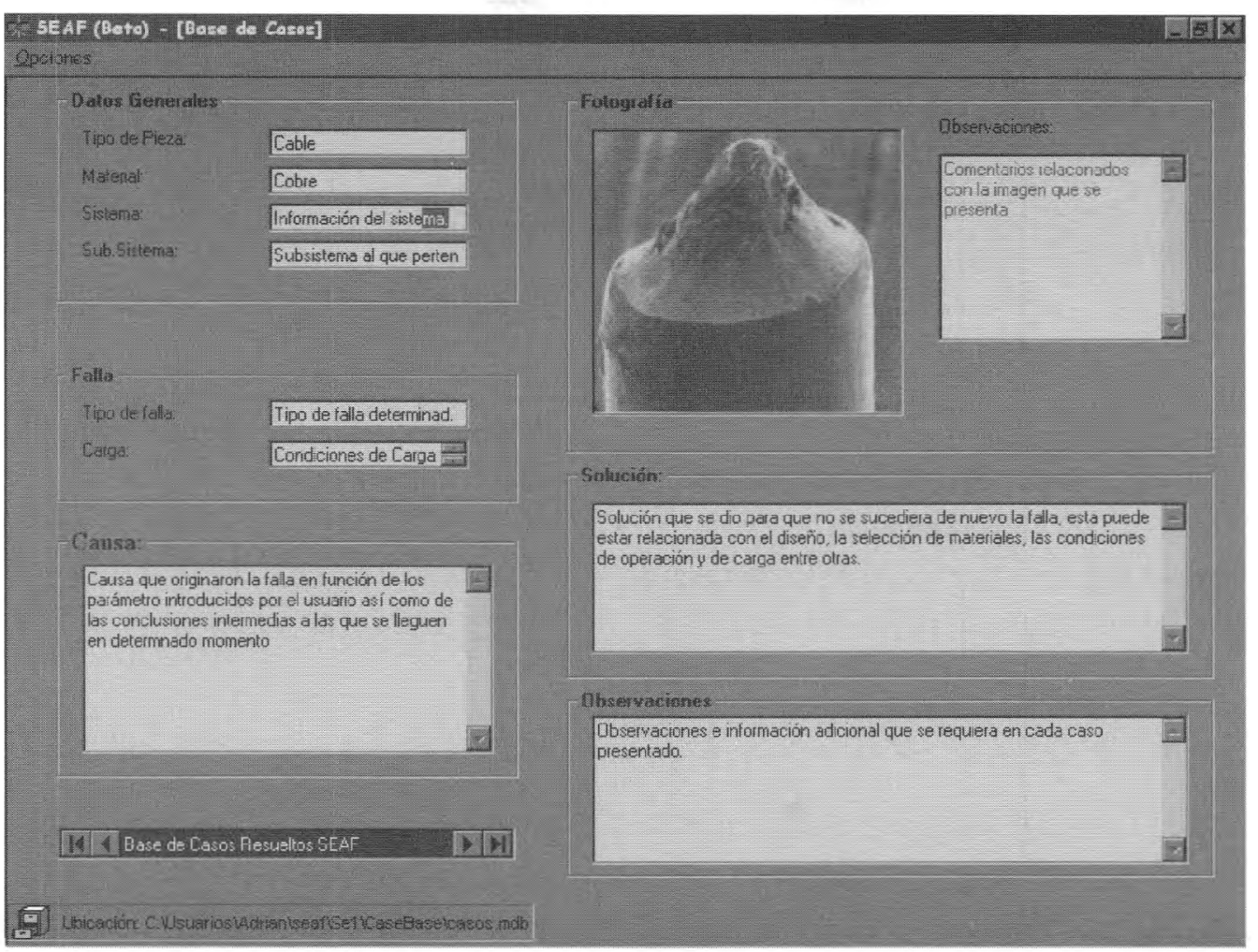

Figura 11. Estructura de un registro de la base de casos

El sistema que se ha desarrollado, busca solucionar casos relativos a fallas de ejes con base en lo que se desprende de la inspección visual sobre el fenómeno, la siguiente etapa sería adicionar una serie de reglas que evalúen aspectos relativos a la información de laboratorio como observaciones metalográficas, mediciones de dureza, etc., con esto se obtendrán mayores niveles de certeza en las conclusiones que se desprendan del análisis.

\section{Conclusiones}

El sistema de análisis de falla de ejes resuelve casos tal y como lo haría el experto humano, garantizado un elevado grado de confiabilidad en los resultados obtenidos.

La estrategia empleada ha permitido avanzar y organizar de una mejor manera las actividades del grupo de desarrollo del sistema.

La experiencia adquirida en la elaboración de este sistema, permite que los módulos de análisis de falla de otros elementos se puedan desarrollar con mayor facilidad para integrar de manera gradual el sistema global de análisis de falla de elementos mecánicos metálicos.

\section{Agradecimientos}

Se reconoce el apoyo económico proporcionado por la Dirección General de Asuntos del Personal Académico de la UNAM, para el proyecto IN 105198 "Sistema experto para el análisis de falla de piezas metálicas mecánicas" .

Se agradece la participación de los tesistas Víctor Eduardo Rosas y Raúl López, quienes colaboraron en la programación de módulos, así como al M.en C. Rafael Schouwenaars por sus aportaciones como experto en análisis de falla.

\section{Referencias}

ASM. (1974). Source Book in Failure Analysis. American Society for Metals.

ASM. (1986). Failure Analysis and Prevention. Metals

Handbook, Vol. X, American Society for Metals ASM. (1987). Fractography and Atlas of Fractographs. Metals Handbook, Vol. IX, American Society for Metals. 
DOI: http://dx.doi.org/10.22201/fi.25940732e.2002.03n3.012

Cerrud S., et al. E. (2000). Adquisición y representación del conocimiento en un sistema experto para análisis de falla. Memorias del VI Congreso anual de la Sociedad Mexicana de Ingeniería Mecánica, Colima, Colima.

Cerrud S., et al. (1998). Propuesta para el desarrollo de un sistema experto en análisis de falla de elementos mecánicos metálicos. Memorias del IV Congreso anual de la Sociedad Mexicana de Ingeniería Mecánica, Ciudad Juárez, Chihuahua.

Dieter G. (1991). Engineering Design, Mc Graw-Hill. Graham I. y Mellor B. (1997 a). Expert \& Knowledge Based Systems in Failure Analysis. Part of the Engineering Materials Failure Series. Southampton, University U.K.
Graham J. y Mellor B. (1997 b). Expert Systems in Failure Analysis. Part of the Engineering Materials Failure Series. Southampton, University UK.

Jacobo V., Cerrud S. y Ortiz A. (2000). Prototipo de un sistema experto para el análisis de falla de elementos mecánicos. Memorias del X Congreso Latinoamericano de Investigación de Operaciones, México, DF.

Roberge P.R. (1996). Failure Analysis 6y Case-Based Reasoning. Proceedings of the international Congress InterCorr/96.

Walton H.W. (1991). Failure Diagnostics- Applications of Expert Systems. Proceedings of the international Conference on Failure Analysis.

\section{Semblanza de los autores}

Víctor Hugo lacobo-Armendáriz. Ingeniero mecánico electricista (industrial) y maestro en ingeniería en el área de investigación de operaciones por parte de la Facultad de Ingeniería, UNAM. Ha participado en proyectos para la industria y es profesor en el área de manufactura y materiales. Actualmente trabaja en un proyecto de investigación doctoral con líneas orientadas al desarrollo de sistemas expertos en análisis de falla.

Sara Mercedes Cerrud-Sánchez. Es ingeniera mecánica electricista (industrial) y maestra en ingeniería mecánica por la Facultad de Ingeniería de la UNAM. Ha participado en más de 25 estudios, proyectos y asesorías para el sector industrial. Fungió como corresponsable en el proyecto Sistema experto para el análisis de falla de piezas mecánicas, metálicas. Tiene más de 25 publicaciones y actualmente es profesora del área de manufactura y materiales.

Marco Antonio Ramírez-Rodríguez. Estudiante de décimo semestre de la carrera de ingeniería mecánica. Participó en el proyecto PAPIT Sistema experto para análisis de falla de piezas mecánicas metálicas. Actualmente se encuentra desarrollando su tesis con el tema Metodología para el desarrollo de un sistema experto en análisis de falla.

Armando Ortiz-Prado. Es ingeniero mecánico electricista y maestro en ingeniería mecánica por la Facultad de Ingeniería, UNAM. En 1989, obtuvo la distinción Universidad Nacional para jóvenes académicos en el área de docencia en ciencias exactas. Ha dirigido más de 70 tesis entre licenciatura y posgrado, así como más de 50 estudios y proyectos para el sector industrial en el área de manufactura, materiales y en particular, análisis de falla. Tiene más de 40 publicaciones relacionadas con el área de su especialización en foros nacionales e internacionales. Actualmente es profesor y coordinador de la Unidad de Investigación y Asistencia Técnica en Materiales. 